\title{
Intervalos biológicos de referencia del perfil lipídico
}

\author{
Biological reference intervals of lipid profile
}

\author{
Yeisson Galvis, Jacqueline Barona, Jaiberth Antonio Cardona \\ - Medellín (Colombia)
}

\section{Resumen}

Introducción: la clasificación de la concentración de cualquier analito como patológico o no implica que el valor de la medición sea comparado con valores de individuos similares, para lo cual es necesario obtener intervalos biológicos de referencia (IBR). Los IBR del perfil lipídico adoptados por los laboratorios clínicos son principalmente los propuestos por las casas comerciales y los obtenidos por los paneles de expertos. Sumado a las limitadas publicaciones sobre los IBR del perfil lipídico para nuestra población, es oportuno precisar que variables como el sexo y la edad pueden estar relacionadas con modificaciones en el metabolismo lipídico.

Objetivo: estimar los intervalos biológicos de referencia del perfil lipídico en una población atendida en un laboratorio de Medellín.

Métodos: estudio descriptivo transversal en 81 individuos sanos; los IBR se estimaron a partir del estadístico $\mathrm{X} \pm\left[\mathrm{Z}_{\alpha / 2} *(\mathrm{DE} / \sqrt{ } \mathrm{n})\right]$, confianza de $95 \%$ y precisión de $5 \%$. En el análisis bivariado se utilizó Anova y la prueba t Student. Todo fue realizado en SPSS 21.0 ${ }^{\circledR}$. En los IBR de hombres y mujeres sólo se hallaron diferencias en el colesterol total y los índices colesterol total/cholesterol HDL y triglicéridos/cholesterol HDL. Analizando los grupos etarios, se hallaron diferencias en los triglicéridos y el cholesterol VLDL siendo menor en los adultos jóvenes en comparación con los adultos medios y mayores; para el índice triglicéridos/cholesterol HDL se halló diferencia entre los adultos jóvenes y mayores.

Conclusión: este estudio permitió determinar los IBR del perfil lipídico, los cuales son importantes para el diseño de estrategias de prevención primaria para dislipidemias en la población estudiada. (Acta Med Colomb 2016; 41: 29-35).

Palabras clave: lípidos, valores de referencia, intervalos de confianza, dislipidemias, triglicéridos, colesterol.

\section{Abstract}

Introduction: the classification of the concentration of any analyte as pathological or not, implies that the measurement be compared to values of similar individuals, for which is necessary to obtain Biological Reference Intervals (BRI). The BRI of lipid profile adopted by clinical laboratories are mainly the offered by commercial houses and those obtained by expert panels. In addition to the limited literature on BRI of lipid profile for our population, it is appropriate to specify that variables such as sex and age may be related to changes in lipid metabolism.

Objective: to estimate the biological reference intervals of lipid profile in a population treated at a laboratory in Medellin.

Methods: descriptive cross-sectional study in 81 healthy individuals; BRI were estimated from statistical $\mathrm{X} \pm[\mathrm{Z} \alpha / 2 *(\mathrm{DE} / \sqrt{ } \mathrm{n})], 95 \%$ confidence and accuracy of $5 \%$. Anova and $\mathrm{t}$ Student test was used in the bivariate analysis. Everything was done in SPSS $21.0^{\circledR}$. In the BRI of men and women differences were only found in total cholesterol levels and total / HDL cholesterol and triglycerides / HDL cholesterol. Analyzing age groups, differences in triglycerides and VLDL-C were found, being these lower in young adults compared with the middle-aged adults and the elderly; for index triglycerides / VLDL cholesterol difference between young and older adults was found.
Dr. Yeisson Galvis Pérez: Microbiólogo y Bioanalista, Servicio Social Obligatorio en Química Clínica, Escuela de Microbiología Universidad de Antioquia; Dra. Jacqueline Barona Acevedo: Bacterióloga y Laboratorista Clínico, MSc., PhD. Nutritional Sciences. Escuela de Microbiología. Universidad de Antioquia; Dr. Jaiberth Antonio Cardona-Arias: Microbiólogo y Bioanalista, MSc Epidemiología. Escuela de Microbiología. Universidad de Antioquia. Facultad de Medicina, Universidad Cooperativa de Colombia. Medellín (Colombia).

Correspondencia. Dr. Jaiberth Antonio Cardona Arias. Medellín (Colombia).

E-mail: jaiberthcardona@gmail.com Recibido: 18/IV/2015 Aceptado: 23/II/2016 
Conclusion: this study allowed determining the BRI of lipid profile, which are important for designing strategies for primary prevention of dyslipidemia in the studied population. (Acta Med Colomb 2016; 41: 29-35).

Keywords: lipids, reference values, confidence intervals, dyslipidemia, triglycerides, cholesterol.

\section{Introducción}

La determinación o clasificación de la concentración de cualquier analito como patológico o no, implica que el valor de la medición sea comparado con valores de individuos similares, para lo cual es necesario obtener intervalos biológicos de referencia (IBR) que permitan determinar fehacientemente el estado de salud del paciente y las implicaciones del resultado obtenido. Los IBR representan valores particulares hallados en una población determinada para fines comparativos (1), cuya muestra de referencia debe ser un grupo representativo de la población (95\%), al cual se le realizan las determinaciones analíticas y con los datos obtenidos se infieren los parámetros de dicha población (2).

En química clínica se han estimado IBR para analitos como la glucosa, urea, ácido úrico, creatinina, colesterol, triglicéridos, bilirrubina, amilasa, gamma glutamil transpeptidasa (GGT), aspartato aminotransferasa (AST), alanina aminotransferasa (ALT), lactato deshidrogenasa, proteínas totales (3-6), leptina, cortisol e insulina (7). Una revisión de los IBR más utilizados en los laboratorios cubanos (8) muestra su variabilidad según la casa comercial que provea los reactivos para las determinaciones, evidenciando la importancia de utilizar IBR propios para cada población. En Colombia se encuentran estudios que determinan IBR en química clínica, pero para para animales, tales como el de Báez, Cabra y Ruiz sobre creatinina en perros (9) y el de Castañeda, Buriticá y Cruz sobre glucosa en la lora común (Amazona ochrocephala) (10). En la búsqueda bibliográfica realizada no se encontraron estudios en Colombia y concretamente en Medellín, sobre IBR para los parámetros del perfil lipídico en humanos.

Los IBR del perfil lipídico adoptados por los laboratorios clínicos en Medellín, son principalmente los propuestos por la casa comercial que comercializa los reactivos para las determinaciones y/o los propuestos por los paneles de expertos (4). Estos IBR generalmente están calculados en poblaciones diferentes a las del área de influencia de las instituciones de salud, por lo que no resultan acordes con el perfil clínico y epidemiológico específico y en consecuencia, pueden ser limitados para orientar acciones de prevención y seguimiento a los pacientes de un lugar en particular (11). Este hecho resulta de mayor relevancia al tener presente que la medición de los lípidos en sangre es parte fundamental para el diagnóstico, tratamiento y seguimiento del riesgo para enfermedades cardiovasculares $(12,13)$, las cuales constituyen la primera causa de muerte en Colombia y en el mundo $(14,15)$.

Sumado a la ausencia de estudios publicados sobre los IBR del perfil lipídico en nuestra población, es importante precisar que variables como el sexo y la edad están relacionadas con modificaciones en el metabolismo lipídico debido a la disparidad hormonal entre hombres y mujeres, y a patrones alimentarios, de actividad física y otros estilos de vida que presentan diferencias crasas por grupo etario y sexo, y que suponen variabilidad en los IBR de algunos subgrupos poblacionales $(16,17)$.

Teniendo en cuanta lo anterior, el objetivo de este estudio fue estimar los IBR del perfil lipídico en una población atendida en un laboratorio de Medellín, 2013.

\section{Tipo de estudio}

\section{Material y métodos}

Descriptivo transversal.

\section{Población, muestra y muestreo}

La investigación se realizó en adultos, de ambos sexos, residentes en el área metropolitana de Antioquia y atendidos durante el año 2013 en un laboratorio de segundo nivel de complejidad de Medellín. A partir de 6384 sujetos atendidos se excluyeron 790 por consumir medicamentos para tratar diversos desórdenes crónicos como dislipidemia, hipertensión, entre otros; 2566 presentaron triglicéridos con valores $\geq 150 \mathrm{mg} / \mathrm{dL}, 1244$ con colesterol total $\geq 200 \mathrm{mg} /$ dL, 655 con colesterol LDL (cLDL; del inglés low-density lipoprotein) $\geq 100 \mathrm{mg} / \mathrm{dL}, 175$ hombres con colesterol HDL (cHDL; del inglés high-density lipoprotein) $<40 \mathrm{mg} /$ $\mathrm{dL}, 153$ mujeres con $\mathrm{cHDL}<50 \mathrm{mg} / \mathrm{dL}$ y 319 con perfil lipídico incompleto.

Con base en los anteriores criterios de inclusión y exclusión se delimitó una población de 482 individuos sanos (según mediciones bioquímicas y consumo de fármacos) a partir de los cuales se seleccionó una muestra aleatoria simple de 81 sujetos con un intervalo de confianza de $95 \%$, de precisión del muestreo de $5 \%$ y desviación estándar 25 en el valor de los analitos del perfil lipídico.

\section{Recolección de la información}

Se utilizó información secundaria consignada en software ENTERPRISE proporcionada por el laboratorio de segundo nivel de complejidad de Medellín, específicamente las variables edad, sexo, triglicéridos, colesterol total, cHDL, cLDL, colesterol VLDL (cVDL; del inglés very low-density lipoprotein) y los índices aterogénicos colesterol total / cHDL, cLDL/cHDL y triglicéridos/cHDL. La edad se categorizó en adultos jóvenes entre 20 y 44 años, adultos medios entre 45 y 64 años y adultos mayores a partir de los 65 años.

Las mediciones del perfil lipídico se realizaron en un laboratorio de segundo nivel con certificación de calidad y 
personal capacitado para el desarrollo de estas pruebas. Se empleó un equipo automatizado Cobas c311 (Roche ${ }^{\circledR}$ ) y métodos enzimáticos colorimétricos. Para la cuantificación de triglicéridos el método es lineal hasta $885 \mathrm{mg} / \mathrm{dL}$ y trazable con espectrometría de masa con dilución isotópica (ID/MS) (18), para colesterol total la linealidad del método es hasta $800 \mathrm{mg} / \mathrm{dL}$ y es trazable con el método Abell/Kendall y con ID/MS (19), para el cHDL el método es lineal hasta 120 $\mathrm{mg} / \mathrm{dL}$ y es trazable con el método de referencia definido por el CDC (Centers for Disease Control and Prevention), según el "protocolo de certificación para los métodos de colesterol HDL para fabricantes" del sistema nacional de referencia para el colesterol de los Estados Unidos (20).

\section{Análisis estadístico}

La descripción del grupo se realizó con proporciones y medidas de resumen. Para describir los parámetros del perfil lipídico se utilizaron medidas de tendencia central, dispersión, posición y forma o distribución; en estas últimas se calculó el coeficiente de asimetría de Fisher y coeficiente de curtosis tomando la distribución como simétrica y mesocúrtica en valores de $0 \pm 0.5$ (21).

Para evaluar si los parámetros del perfil lipídico cumplían el supuesto de normalidad se realizó la prueba Kolmogorov-Smirnov con corrección de la significación de Lilliefors, en caso que los datos no cumplieran este supuesto se realizó transformación por la función potencial de Box Cox, la cual presenta la ventaja sobre otras transformaciones de corregir tanto asimetrías positivas como negativas, en éstas se corroboró el supuesto de normalidad y se realizó reconversión de los datos para el cálculo de los IBR (2).

La identificación de los valores extremos y atípicos (aberrantes) se realizó con el método gráfico a través del diagrama de cajas, el cual se complementó con la prueba de Grubbs (Desviación extrema estudentizada), en este último se estableció un valor crítico $\mathrm{Z}$ de 3.31 para una tamaño de muestra de 81 y se calculó el valor $\mathrm{Z}$ con su valor $\mathrm{p}(\mathrm{Vp})$ para cada observación, si el valor $\mathrm{Z}$ superaba el valor crítico y el $\mathrm{Vp}$ resultaba menor a 0.05 se concluía que el dato era atípico y se eliminaba de los análisis posteriores; todo ello bajo la hipótesis nula que plantea que en la muestra no existen datos atípicos (22). Cabe aclarar que en la identificación de valores atípicos no se empleó el estadístico de Dixon dado que éste sólo presenta buena potencia para tamaño de muestra entre 4 y 30 (23) y que ambos estadísticos constituyen pruebas paramétricas que suponen una distribución normal $(22,24,25)$.

Una vez corroborado el supuesto de normalidad y eliminar posibles datos atípicos, se estimaron los IBR del 95\% para la media de los parámetros del perfil lipídico a partir del estadístico $\mathrm{X} \pm\left[\mathrm{Z}_{\alpha / 2} *(\mathrm{DE} / \sqrt{ } \mathrm{n})\right]$ ya que éste aumenta la precisión de la estimación (26).

Una vez estimados los IBR de la población de referencia se estimaron los IBR según sexo y grupo etario, en el análisis bivariado se compararon los IBR de la población general frente a los hallados en cada subgrupo (hombre, mujer, adulto medio, adulto joven y adulto mayor) a través de la prueba t Student para muestras independientes, ello con el fin de explorar la variabilidad de cada uno de estos subgrupos frente a la población general. Se compararon los IBR de hombres y mujeres a través de la prueba de $t$ Student y de los tres grupos etarios entre sí por medio de Anova, evaluando el cumplimiento del supuesto de normalidad bivariada con la prueba Shapiro-Wilk y de homocedasticidad con Levene. Además se estableció el grado de correlación entre los parámetros del perfil lipídico a través del estadístico de Pearson. $21.0^{\circledR}$.

Se utilizó una significación estadística de 0.05 en SPSS

\section{Resultados}

El 59.3\% $(\mathrm{n}=48)$ fueron mujeres y $40.7 \%(\mathrm{n}=33)$ hombres; $28.4 \%(\mathrm{n}=23)$ adultos jóvenes, $25.9 \%(\mathrm{n}=21)$ adultos medios y $45.7 \%(\mathrm{n}=37)$ adultos mayores. La edad

Tabla 1. Descripción de los valores del perfil lipídico del grupo de estudio.

\begin{tabular}{|c|c|c|c|c|c|c|}
\hline $\begin{array}{l}\text { Parámetros del perfil } \\
\text { lipídico }(\mathrm{mg} / \mathbf{d L})\end{array}$ & Media \pm DE & Mediana (RI) & Rango & Asimetría & Curtosis & Vp K-S \\
\hline Triglicéridos (Tg) & $105 \pm 26.4$ & $109.6(92.7-126.3)$ & $39.4-145.6$ & -0.724 & -0.206 & 0.053 \\
\hline Colesterol total (CT) & $157.3 \pm 21.8$ & $161.1(142.6-173.4)$ & 108.7-197.9 & -0.477 & -0.298 & $0.200^{*}$ \\
\hline Colesterol LDL (cLDL) & $77.8 \pm 16.3$ & $82.2(65.3-90.6)$ & $35.3-99.5$ & -0.765 & -0.342 & 0.0 \\
\hline Colesterol VLDL (cVLDL) & $21 \pm 5.28$ & $21.9(18.5-25.2)$ & $7.9-29.1$ & -0.724 & -0.206 & 0.053 \\
\hline Colesterol HDL (cHDL) & $58.5 \pm 13.9$ & $54.1(50.1-63.5)$ & 41-104.4 & 1.408 & 2.065 & 0.0 \\
\hline Colesterol no HDL & $98.8 \pm 17.6$ & $103.9(84.6-111.5)$ & $50.2-127.8$ & -0.766 & -0.080 & 0.004 \\
\hline \multicolumn{7}{|l|}{ Índices } \\
\hline $\mathrm{CT} / \mathrm{cHDL}$ & $2.77 \pm 0.50$ & $2.75(2.41-3.11)$ & $1.71-3.87$ & 0.205 & -0.540 & $0.200^{*}$ \\
\hline cLDL / cHDL & $1.39 \pm 0.41$ & $1.35(1.05-1.67)$ & $0.60-2.23$ & 0.168 & -0.693 & $0.200^{*}$ \\
\hline $\mathrm{Tg} / \mathrm{cHDL}$ & $1.90 \pm 0.66$ & $1.88(1.43-2.36)$ & $0.55-3.34$ & 0.024 & -0.466 & $0.200^{*}$ \\
\hline
\end{tabular}


media fue de $56 \pm 20.5$ años, rango intercuartil de 38.5 a 71.6 y rango entre 20 y 90.

En la Tabla 1 se presentan las medidas de resumen de los parámetros del perfil lipídico, en éste se hallaron coeficientes de variación entre $13.9 \%$ (colesterol total) y $25.1 \%$ (trigliceridos y cVLDL) para los analitos, mientras que en los índices fueron $18.0 \%$ para colesterol total/cHDL, $29.5 \% \mathrm{cLDL} / \mathrm{cHDL}$ y $34.7 \%$ para triglicéridos/cHDL. El $50 \%$ de los sujetos presentó valores de $109.6 \mathrm{mg} / \mathrm{dL}, 161.1$ $\mathrm{mg} / \mathrm{dL}, 82.2 \mathrm{mg} / \mathrm{dL}, 21.9 \mathrm{mg} / \mathrm{dL}$ y $54.1 \mathrm{mg} / \mathrm{dL}$ o menos en los triglicéridos, colesterol total, cLDL, cVLDL y cHDL, respectivamente.

Se hallaron distribuciones simétricas para el colesterol total y los tres índices evaluados, los demás parámetros presentaron asimetría negativa, con excepción del cHDL; los triglicéridos, colesterol total, cLDL, cVLDL, el colesterol no HDL y el índice triglicéridos/cHDL presentaron una distribución mesocúrtica, las demás, con excepción del cHDL fueron platicúrticas. Además, la mayoría de parámetros del perfil lipídico presentaron una distribución normal, con excepción del cLDL, cHDL y el colesterol no HDL, los cuales fueron normalizados según se indicó en la metodología (Tabla 1).

En la identificación de valores atípicos con el método gráfico se presentaron resultados positivos para los triglicéridos, cVLDL y cHDL; sin embargo, con el método analítico según la prueba de Grubbs, ningún parámetro presentó datos atípicos dado que todos los valores $\mathrm{Z}$ fueron inferiores al valor crítico de 3.31 con los subsecuentes $\mathrm{Vp}$ mayores a 0.05 (Figura 2).

En la Tabla 2 se presentan los IBR para la población general y desagregados según sexo y grupo etario, con base en la comparación de sus límites se observa que, frente a los valores de la población general, las mujeres presentaron valores estadísticamente iguales en los parámetros del perfil lipídico ( $\mathrm{Vp}>0.05)$, en los hombres se hallaron IBR menores en colesterol total $(\mathrm{Vp}<0.05)$ y cHDL $(\mathrm{Vp}<0.05)$, y mayores en el índice trigliceridos/cHDL ( $\mathrm{Vp}<0.05)$; por su parte, los adultos jóvenes presentaron valores menores en triglicéridos $(\mathrm{Vp}<0.05)$ y cVLDL $(\mathrm{Vp}<0.05)$, mientras

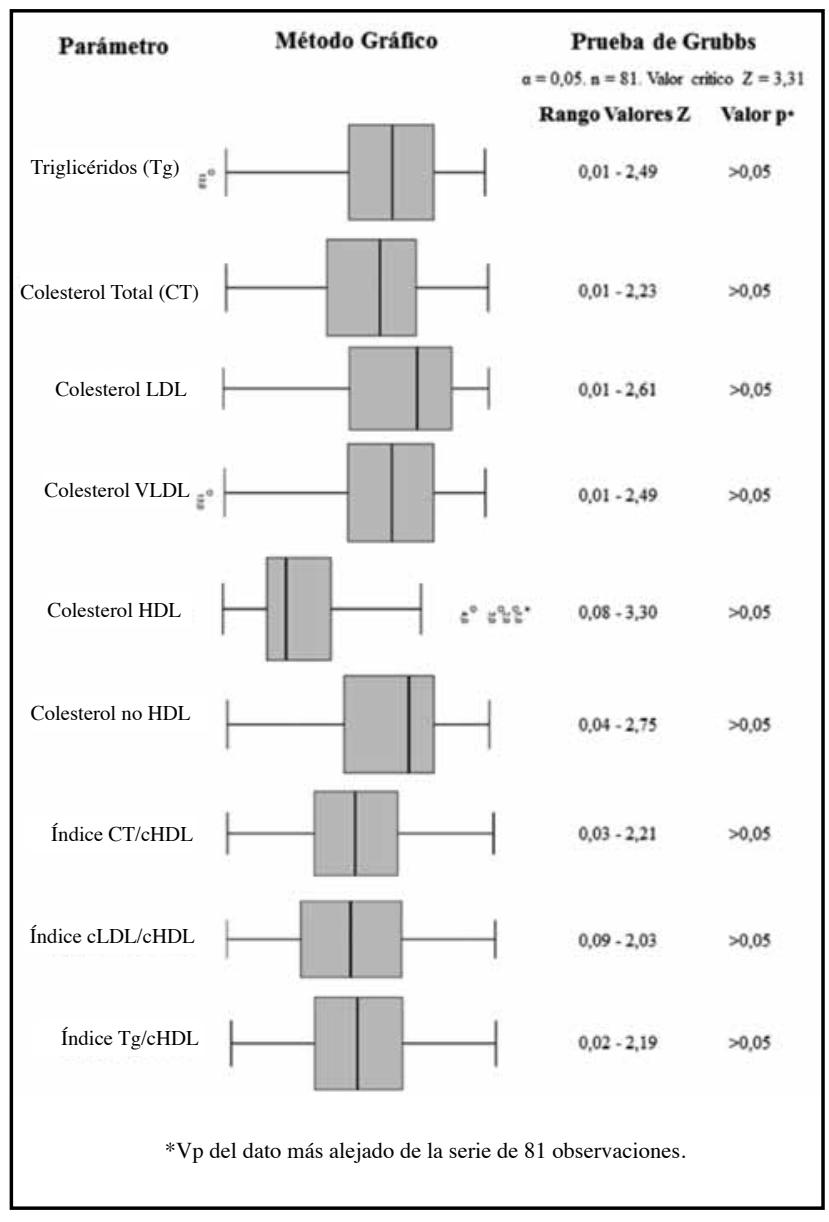

Figura 2. Identificación de valores atípicos en los parámetros del perfil lipídico por el método gráfico y la prueba de Grubbs.

que los IBR en los adultos medios y adultos mayores fueron estadísticamente iguales a los de la población total (Tabla 2).

En las comparaciones entre los IBR de hombres y mujeres sólo se hallaron diferencias en el colesterol total $(\mathrm{Vp}$ $\mathrm{t}$ Student $=0.000 . \mathrm{IC}=12.7 ; 30.0), \mathrm{cHDL}(\mathrm{Vp} \mathrm{t}$ Student $=$ $0.000 . \mathrm{IC}=11.2 ; 20.6)$, índice colesterol total/cHDL (Vp t

Tabla 2. Intervalos biológicos de referencia del perfil lipídico en la población de estudio y específicos según sexo y grupo etario.

\begin{tabular}{|c|c|c|c|c|c|c|}
\hline \multirow[t]{2}{*}{ Parámetro } & \multirow[t]{2}{*}{ Población global } & \multicolumn{2}{|c|}{ Específico por sexo } & \multicolumn{3}{|c|}{ Específico por grupo etario } \\
\hline & & Mujeres & Hombres & Adulto joven & Adulto medio & Adulto mayor \\
\hline Triglicéridos (Tg) & $99.2-110.9$ & $94.8-110.9$ & $99.6-116.9$ & $72.2-98.9$ & $95.7-118.2$ & $110.7-121.4$ \\
\hline Colesterol total & $152.5-162.1$ & $161-171.1$ & $137-152.3$ & $142.3-162.9$ & $157-172.4$ & $148.5-163.5$ \\
\hline cLDL & $74.2-81.4$ & $76.5-84.4$ & $67.1-80.7$ & $69.3-85$ & $74.4-86.3$ & $71-82.4$ \\
\hline cVLDL & $19.8-22.2$ & $18.9-22.2$ & $19.9-23.4$ & $14.4-19.8$ & $19.1-23.6$ & $22.1-24.3$ \\
\hline cHDL & $55.4-61.6$ & $61.1-68.9$ & $46.4-51.8$ & $52.5-64.2$ & $55.3-70.7$ & $52.1-60$ \\
\hline Colesterol no HDL & $94.9-102.7$ & $97-105.1$ & $87.8-103.3$ & $85.7-102.8$ & $95.1-108.4$ & $94-105.8$ \\
\hline $\mathrm{CT} / \mathrm{cHDL}$ & $2.66-2.88$ & $2.51-2.72$ & $2.80-3.20$ & $2.47-2.92$ & $2.49-2.98$ & $2.69-2.99$ \\
\hline cLDL/cHDL & $1.30-1.48$ & $1.19-1.38$ & $1.37-1.72$ & $1.19-1.57$ & $1.17-1.57$ & $1.28-1.54$ \\
\hline $\mathrm{Tg} / \mathrm{cHDL}$ & $1.75-2.04$ & $1.49-1.81$ & $2.04-2.26$ & $1.25-1.86$ & $1.52-2.12$ & $1.98-2.33$ \\
\hline
\end{tabular}




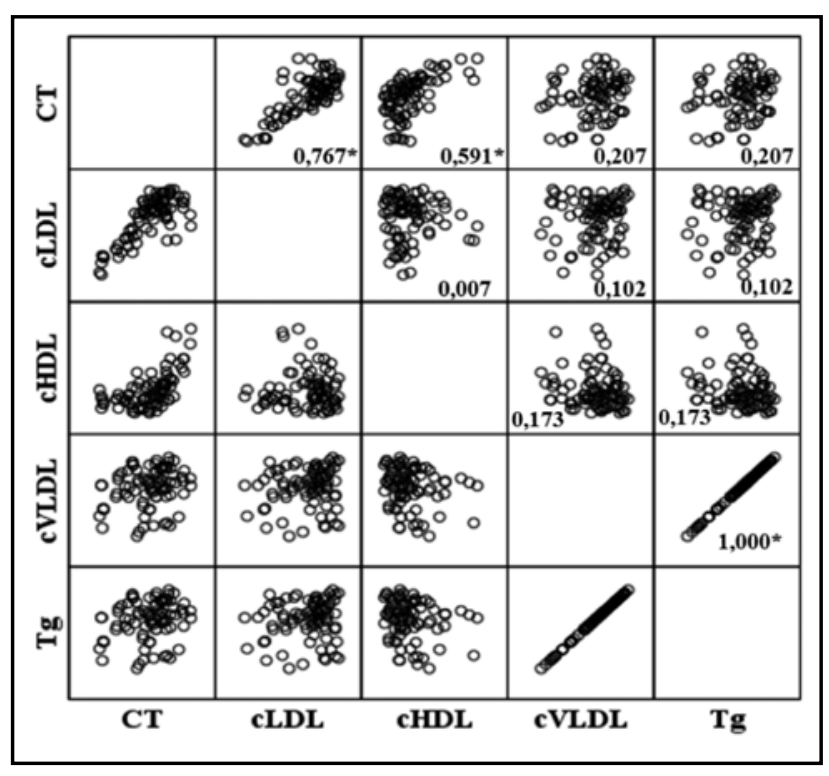

Figura 2. Coeficientes de correlación de Pearson entre las fracciones del perfil lipídico $(* V p=000)$.

Student $=0.001 . \mathrm{IC}=-0.61 ;-0.15)$ y el índice triglicéridos $/$ cHDL (Vp t Student $=0.000$. IC $=-0.87 ;-0.34$ ).

En las comparaciones por grupo etario para los IBR de triglicéridos ( Vp Anova $=0.000)$ no se observaron diferencias significativas entre adultos medios y mayores, pero fue menor el IBR en los adultos jóvenes en comparación con los adultos medios (Vp HSD Tukey $=0.009$. IC $=-38.2 ;-4.6$ ) y adultos mayores (Vp HSD Tukey $=0.000 . \mathrm{IC}=-45.4 ;-15.8$ ). Se observaron hallazgos similares para los IBR de cVLDL $($ Vp Anova $=0.000)$ y de triglicéridos en estos grupos etarios. Para el índice triglicéridos/cHDL (Vp Anova $=0.002$ ) sólo se halló diferencia entre los adultos jóvenes y los mayores (Vp HSD Tukey $=0.001 . \mathrm{IC}=-1.0 ;-0.2$ ).

Entre las fracciones del perfil lipídico sólo se registraron correlaciones positivas y estadísticamente significativas entre los triglicéridos y el cVLDL (como era de esperarse), y el colesterol total con el cLDL y el cHDL, respectivamente (Figura 2).

\section{Discusión}

Los IBR de la población de estudio resultaron mayores para el cHDL y menores en colesterol total, triglicéridos y cLDL, en comparación con los valores propuestos por la ATP-III (Adult Treatment Panel III) (27) y que son los más utilizados en el ámbito clínico como criterio diagnóstico de dislipidemias; esto resulta relevante en términos de prevención primaria en la población de estudio, dado que con los IBR estimados se pueden identificar personas con valores superiores o inferiores (para el caso de cHDL) a los de su población, previo al desarrollo de una dislipidemia.

Los puntos de corte propuestos por la ATP-III se basan en una revisión sistemática de la literatura por parte de un grupo de expertos (28), con el propósito que sirvan de cri- terio diagnóstico; esta claridad es relevante dado que con ello se delimitan dos ámbitos de uso de los IBR, el primero en el cual se evalúa y determina los puntos de corte basados en la evidencia para que un IBR sea diagnóstico, como en el caso de las guías de la ATP, y el expuesto en este estudio en el que los IBR no se toman como criterio diagnóstico sino como una detección temprana de alteración, según los valores estimados como "normales o reales" para la población de la cual proviene el sujeto de estudio.

En relación con los análisis desagregados por sexo y grupo etario, se halló que los adultos jóvenes presentaron un IBR menor tanto para triglicéridos como para cVLDL comparado con los IBR de la población general. No hubo diferencias significativas por sexo para estos parámetros y el IBR de los triglicéridos fue más alto en adultos mayores con respecto a los adultos jóvenes. Estos resultados tienen sustento en la asociación estrecha que existe entre los triglicéridos y el cVLDL a razón de su metabolismo $(29,30)$. La diferencia entre los IBR en el interior de los grupos etarios podría explicarse porque en el adulto mayor, como parte del proceso de envejecimiento, se incrementa su masa grasa, principalmente a nivel central y disminuye su gasto energético, lo cual aumenta los niveles de triglicéridos en la sangre y consecuentemente las producción de lipoproteínas VLDL $(31,32)$.

Aunque el IBR para colesterol total en hombres difirió (fue menor) del estimado para las mujeres y para la población general, el IBR para el cHDL fue considerablemente menor. Esto último ocasionando mayores valores en los índices aterogénicos estudiados en los hombres, lo que podría suponer una mayor exposición de éstos a diversos factores de riesgo relacionados con dislipidemias y estilos de vida, como se ha documentado en investigaciones previas que refieren un mayor consumo de cigarrillo y alcohol en hombres colombianos (16); aunado a las diferencias hormonales, las cuales influencian el metabolismo lipídico (33).

El IBR para el índice triglicéridos/cHDL en hombres fue significativamente mayor al IBR de la población general, y el de las mujeres, mientras que el IBR para el índice colesterol total/cHDL sólo fue diferente entre hombres y mujeres; esto podría atribuirse, al igual que lo descrito previamente, a procesos metabólicos y exposición a factores de riesgo diferenciales entre hombres y mujeres $(16,33)$. El índice triglicéridos/cHDL, que es predictor de la resistencia a la insulina (34), fue diferente entre el adulto joven y el adulto mayor, siendo mayor en este último grupo etario, lo cual podría estar relacionado con los procesos de envejecimiento y/o cambios en el estilo de vida en el adulto mayor (ej. mayor sedentarismo) mencionado en párrafos anteriores (32), principalmente el aumento de la grasa corporal de tipo visceral, puesto que la resistencia a la insulina tiene relación estrecha con el incremento de la obesidad central $(35,36)$.

Por otra parte, se debe considerar que los IBR estimados no están dados para delimitar condiciones que requieran tratamiento dado que éstos definen los límites entre los 
cuales oscila la media poblacional o real a partir de los datos de la muestra seleccionada; de ello se derivan varias aplicaciones: i) comparar poblaciones, ii) detectar diferencias entre los IBR de varias poblaciones con el fin de establecer si tales diferencias podrían respaldar recomendaciones de intervención clínica, iii) permitir una mejor interpretación de resultados en la clínica o programas de control $(37,38)$, iv) evaluar la posibilidad de extrapolar los valores a poblaciones diferentes, v) evaluar la efectividad de una determinación analítica o para evaluar los efectos de medicamentos u otras intervenciones sobre las determinaciones bioquímicas, vi) comparar valores aislados con los IBR estimados para la población (2).

Los valores de referencia o IBR pueden desagregarse según diferentes criterios como: i) estimación poblacional o individual, ii) estimación univariada o multivariada, y iii) especificados o no especificados en el tiempo (estos últimos aluden a valores de varios sujetos sin incluir en los análisis sus ritmos o variaciones biológicas) (2); en la presente investigación se estimaron IBR poblacionales, univariados y no especificados en el tiempo dada la finalidad de mejorar programas de prevención primaria.

En este orden de ideas, cabe precisar que la estimación de los IBR por métodos multivariados se ha empleado para perfiles de pruebas que dan cuenta del estado o funcionalidad de un órgano específico, aunque en éste se pueden presentar dos paradojas: i) el perfil es normal en la estimación multivariada pero uno o más resultados individuales son "patológicos" (fuera de su IBR), en cuyo caso las desviaciones univariadas resultan de interés clínico. En este caso se deben buscar las razones para la "normalidad" de las estimaciones multivariadas; ii) las estimaciones univariadas son normales y la multivariadas "patológicas", lo que se puede explicar por correlaciones entre las variables individuales. Ante estas paradojas se recomienda su interpretación simultánea (univariada y multivariada) para aumentar la eficiencia del diagnóstico, recabando el hecho que las estimaciones multivariadas presentan menos susceptibilidad a cambios de un analito individual y permiten evidenciar anormalidades que no se podrían detectar con análisis individuales (2).

Los valores de referencia aluden el hallazgo de un analito en un sujeto de referencia, el cual pertenece a la comunidad de referencia del laboratorio y presenta un buen estado de salud según la definición que tome el investigador. En este sentido, se debe precisar que la definición de persona sana en este estudio presenta limitaciones relacionadas con el hecho de no hacer explícitos estados fisiológicos (ejemplo la gestación, lactancia o presión sanguínea) o patológicos subyacentes en la delimitación de la población de referencia (condiciones patológicas o intervenciones médicas), factores de riesgo como obesidad, alcoholismo y tabaquismo; dado que sólo se tuvo presente la información consignada en la base de datos. Sin embargo, debe aclararse que esto no afecta la validez de los resultados ya que los criterios de inclusión y exclusión tomaron en cuenta alteraciones directas del perfil evaluado y la historia farmacológica; además, los estados de salud absolutos no existen por lo que todas las investigaciones relacionadas con IBR presentan definiciones flexibles sobre el estado de salud, lo que afianza la necesidad de fomentar este tipo de estudios en poblaciones específicas y no tomar datos generales de guías establecidas para poblaciones con características fisiológicas, anatómicas, demográficas y epidemiológicas diferentes (2).

Otra posible limitación de este estudio está relacionada con el cálculo de los IBR por subgrupos en los cuales no se emplearon criterios técnicos como el de Harris y Boyd, el de Lathi o el de Linton, sino criterios clínicos y epidemiológicos relacionados con la importancia de los IBR por sexo y grupo etario para orientar acciones en salud y por el hecho que estos subgrupos, presentan diferencias en la exposición a factores de riesgo para alteraciones del metabolismo lipídico (2). Por lo anterior, los IBR específicos para estas dos variables deben tomarse como exploratorios y como línea de base para estudios posteriores.

Entre las ventajas de los IBR estimados se destacan: i) cumplir los requisitos básicos de las fases preanalítica, analítica y posanalítica, como la delimitación de la población de referencia, el cumplimiento de un ayuno mínimo, estandarización en las fases de obtención y procesamiento de la muestra, utilización de los mismos instrumentos analíticos en las determinaciones del perfil y tener control de calidad interno y externo, ii) tomar dos variables clave en la estructura poblacional, como la edad y el sexo, como criterio de partición o cálculo de IBR específicos, iii) utilización de procedimientos estadísticos robustos para la detección y manejo de posibles datos aberrantes (que reflejan errores analíticos o de ejecución) (2). Lo anterior permite aseverar que la dispersión hallada en los IBR de este estudio son las propias de la variabilidad intra e interindividuales de la población, dado que se controlaron las demás fuentes de variabilidad, es decir, la variación atribuible al error analítico (procedimientos utilizados) y al error de ejecución (problemas de selección de los sujetos sanos).

Aunado a lo anterior, se realizó la estimación de los IBR por el método Intervalo de predicción, el cual estima los dos valores límites de la muestra seleccionada entre los cuales se encuentra el valor de referencia en la población de estudio, esta estimación paramétrica de los IBR resulta más sensible y precisa que los cálculos no paramétricos. No se realizó determinación por fractiles ya que éstos representan una parte de la población pero no permiten estimar el valor "real", lo que deriva en una mayor imprecisión en la estimación, además las estimaciones no paramétricas deben incluir como mínimo un tamaño de muestra de 120 sujetos $(1,2)$.

\section{Conclusión}

Este estudio permitió determinar los IBR del perfil lipídico para la población atendida en el laboratorio de la IPS Universitaria-Escuela de Microbiología de Medellín, los cuales son importantes para la realización de prevención 
primaria en la población estudiada y evitar el aumento de dislipidemias. Las diferencias presentadas entre los IBR de la población general con respecto a la desagregación por sexo y grupo etario, evidencian la importancia de implementar medidas de tamización y seguimiento diferenciales por subgrupos, y de realizar más investigaciones en esta área.

\section{Agradecimientos}

Al Laboratorio docente asistencial e investigativo de la IPS Universitaria-Escuela de Microbiología-Universidad de Antioquia por la contribución de los datos.

\section{Conflicto de intereses}

Ninguno de los autores declara conflicto de intereses para la publicación de este manuscrito.

\section{Referencias}

1. Arderiu X. Intervalos de referencia biológicos1. Noticonaquic. 2011; 54: 46-51.

2. Sociedad Española de Bioquímica Clínica y Patología Molecular (SEQC). Valores de referencia. Curso de estadística para el laboratorio Clínico. [Internet]. 2007. [Citado el 12 de diciembre de 2014]. Módulo 3; 1-35. Disponible en: http:// www.seqc.es/es/Varios/7/39/Modulo_3:_Valores_de_referencia/

3. Deveze M, Sanchez D, Cuellar P, Jiménez G, Duran E. Determinación de valores de referencia en bioquímica clínica en suero de derechohabientes de la clínica hospital "B" de Guanajuato. Cont quím. 2011; 6: 3-7.

4. Fuentes G, Diaz P, Hernández R, Cervantes D, Presno J, Alcántara L. Determinación de intervalos de referencia para química clínica en población Mexicana. Rev Latinoamer Ptol Clin. 2013; 60: 43-51.

5. René W, Bonneau G, Castillo M, Juárez M, Cardozo J. Valores de referencia y prevalencia de las alteraciones del perfil lipídico en adolescentes. Arch Argent Pediatr. 2010; 108: 107-15.

6. Mendoza L, Gómez T, Pérez L, Mollineda A, Salazar L, Hernandez V. Perfil bioquímico y valores de referencia en sangre del cordón umbilical. Acta Bioquím Clín Latinoam. 2014; 48: 311-7.

7. Koester T, Valtueña J, Breidenassel C, Beghin L, Plada M, Moreno S, et al. Reference Values for Leptin, Cortisol, Insulin and Glucose, Among European Adolescents and Their Association With Adiposity: the Helena Study. Nutr Hosp. 2014; 30: 1181-90.

8. Edelby L. Valores de referencia del laboratorio más empleados en Cuba. Año 2011.GME. 2011; 13: sin p.

9. Báez P, Cabra C, Ruiz C. Standardization of Serum Creatinine Levels in Healthy Dogs Related to Body Weight at the South Valley of Aburra, Colombia. Rev Med Vet. 2014; 27: 33-40.

10. Castañeda F, Buriticá E, Cruz L. Valores de referencia para hematocrito, hemoglobina, glucosa y electrolitos de la lora común Amazona ochrocephala (Gmelin,1788) cautivos en Ibagué. Orinoquia. 2012; 16: 67-77.

11. Molina K, Vargas E, Tavera S, Pérez R, Mantilla C, Cardona J. Intervalos biológicos de referencia del hemograma en personas sanas, Medellín, 2012. Med Lab. 2013; 19: 267-81.

12. Reiner Z, Catapano A, De Backer G, Graham I, Taskinen M, Wiklund O, et al. Guía de la ESC/EAS sobre el manejo de las dislipemias 2011. Rev Española Cardiol. 2011; 64: 1168.e1-1168.e60.

13. Stone NJ, Robinson J, Lichtenstein AH, Bairey Merz CN, Lloyd-Jones DM, Blum CB, et al. 2013 ACC/AHA Guideline on the Treatment of Blood Cholesterol to Reduce Atherosclerotic Cardiovascular Risk in Adults: A Report of the American College of Cardiology/American Heart Association Task Force on Practice Guidelines. J Am Coll Cardiol. 2013; 63: 2889-2934.

14. Instituto Nacional de Salud (INS). Primer Informe Observatorio Nacional de Salud (ONS). Aspectos relacionados con la frecuencia de uso de los servicios de salud, mortalidad y discapacidad, 2011. Bogotà: Imprenta Nacional de Colombia . 2013. $191 \mathrm{p}$.

15. Mendis S, Puska P, Norrving B. Global Atlas on cardiovascular disease prevention and control. Geneva: World Health Organization, World Heart Federation, World Stroke Organization. 2011.p. 156.

16. Organización Panamericana de la Salud, Ministerio de la Protección Social, Instituto Nacional de Salud, Instituto Colombiano de Bienestar Familiar, Departamento
Administrativo Nacional de Estadística, Coldeportes, et. al. Encuesta Nacional de la Situación Nutricional en Colombia 2010 ENSIN. [Internet]. 2010. [Citado el 21 de enero de 2015]. Disponble en: http://www.icbf.gov.co/portal/page/portal/ Descargas1/Resumenfi.pdf

17. Serna B, Marquez J. Efecto de los factores de riesgo cardiovasculares modificables sobre los niveles de HDL en Medellín. [tesis de maestría] Medellín: Facultad de Medicina, Departamento de Epidemiología, Universidad CES. 2009. 86 p.

18. ROCHE. Instructivo TRIGL: Triglycerides, sistemas cobas c. 2008 p. 3 . Report No.: 2008-11, V 5.

19. ROCHE. Instructivo CHOL2: Cholesterol Gen.2, sistemas cobas c. 2009 p. 3 Report No.: 2009-12, V 6.

20. ROCHE. Instructivo HDLC3: HDL-Cholesterol plus 3rd gereration, sistemas cobas c. 2009 p. 4. Report No.: 2009-03, V 5.

21. Martinez A. Medidas de Distibución - Asimetría y Curtosis. [Internet]. 2007. [Citado el 14 de enero de 2015]. Capitulo V; 1. Disponible en: http://www.spssfree. com/spss/analisis $3 . h t m l$

22.GraphPad Software. Detecting outliers with Grubbs' test. [Internet]. 2010 [Citado el 14 de enero de 2015]. Disponible en: http://graphpad.com/support/ faqid/1598/

23. StatPoint, Inc. Identificación de valores atípicos. Statgraphics. [Internet]. 2007. [Citado el 14 de enero de 2015].25:1-15. Disponible en: http://www.statgraphics. net/wp-content/uploads/2011/12/tutoriales/Identificacion $\% 20 \mathrm{de} \% 20$ Valores $\% 20$ Atipicos.pdf

24. Murphy T, Lau A. Manejo de valores atípicos. Como se evalúa un valor aberrante o inconsistente único. Standardization News. [Internet]. 2008. [Citado el 14 de enero de 2015]. Disponible en: http://www.astm.org/SNEWS/SPANISH/SPND08/ datapoints_spnd08.html

25. Bzik T, Neubauer D. Prueba de valores atípicos. Consideraciones prácticas y filosóficas. Standardization News. [Internet]. 2011. [Citado el 14 de enero de 2015]. Disponible en: http://www.astm.org/SNEWS/SPANISH/SPJF11/datapoints_spjf11.html

26. Miller J, Miller J. Estadística y quimiometría para química analítica. 4 ed. Maté C, Izquierdo R. Traductor. Madrid: Prentice Hall. 2005. 286 p.

27. National Cholesterol Education Program, Adult Treatment Panel III. Executive Summary of the Third Report of the National Cholesterol Education Program (NCEP) Expert Panel on Detection, Evaluation, and Treatment of High Blood Cholesterol in Adults (Adult Treatment Panel III). J Am Med Assoc. 2001. 16; 285: 2486-97.

28. National Institutes of Health, National Cholesterol Education Program Adult Treatment Panel III. Third Report of the National Cholesterol Education Program (NCEP) Expert panel on detection, evaluation, and treatment of high blood cholesterol in adults (Adult treatment panel III). U.S.: Department of health and human services. 2002. $284 \mathrm{p}$.

29. Errico L, Chen X, Martin J, Julve J, Escolà J, Blanco F. Mecanismos básicos : estructura, función y metabolismo de las lipoproteínas plasm. Clin Invest Arter. 2014; 25: 98-103.

30. Bays HE, Toth PP, Kris-etherton PM, Abate N, Aronne LJ, Brown WV, et al. Obesity , adiposity, and dyslipidemia: A consensus statement from the National Lipid Association. J Clin Lipidol. 2013; 7: 304-83.

31. Ruiz A, León Y, Burgos D, Sarduy J, Linares O, Hernández D. Alteraciones de metabolismo lipídico en adultos mayores de 60 años con enfermedades crónicas no transmisibles. Mex Patol Clin. 2009; 56: 36-44.

32. Sociedad Española de Geriatría y Gerontología. Nutrición en el anciano. Guía de buena práctica clínica en geriatría. Madrid: NestleHealthScience. 2013.73 p.

33. Wang X, Magkos F, Mittendorfer B. Sex differences in lipid and lipoprotein metabolism: it's not just about sex hormones. J Clin Endocrinol Metab. 2011; 96: 885 .

34. Oliveira AC, Oliveira AM, Oliveira N, Oliveira A, Almeida M, Veneza LM, et al. Is triglyceride to high-density lipoprotein cholesterol ratio a surrogates for insulin resistance in youth ?. Health. 2013; 5: 481-5.

35. Manzur F, Alvear C. Alayón A. Adipocitos, obesidad visceral, inflamación y enfermedad cardiovascular. Rev Colomb Cardiol. 2010; 17: 207-13.

36. García E. Obesidad, tejido adiposo y resistencia a la insulina. Acta Bioquím Clín Latinoam. 2012; 46: 183-94.

37. Candia R, Caiozzi G. Intervalos de confianza. Rev Méd Chile. 2005; 133: 1111-5.

38. Wayne D. Daniel Wayne W. Bioestadística: Base para el análisis de las ciencias de la salud. 4 ed. Mexico: Editorial Limusa. 2002. 915 p. 